\title{
Electric control of straight stripe conductive mixed-phase nanostructures in La-doped $\mathrm{BiFeO}_{3}$
}

\author{
Kwang-Eun Kim ${ }^{1}$, Byung-Kweon Jang ${ }^{1}$, Yooun $\mathrm{Heo}^{2}$, Jin Hong Lee ${ }^{1}$, Myoungho Jeong ${ }^{3,4}$, Jeong Yong Lee ${ }^{3,4,5}$, \\ Jan Seidel ${ }^{2}$ and Chan-Ho Yang ${ }^{1,5}$
}

This study examines the atomic force microscope (AFM) tip-based electrical formation of tens of microns long stripe (and $\sim 100 \mathrm{~nm}$ wide) inorganic one-dimensional nanostructures based on the morphotropic phase boundary of La-doped $\mathrm{BiFeO}_{3}$ epitaxial thin films. The substitution of Lanthanum into bismuth ferrite not only produces the formation of straight stripe mixedphase patterns but also improves the spatial continuity drastically by two orders of magnitude. We create, switch and erase stripe nanostructures in a reversible and deterministic way. We demonstrate that electrically formed areas with a nearly single variant alignment can be overwritten with different alignments repeatedly, which reflects the reversible and nonvolatile nature of the switching process. In addition, we explore the functionality of the created nanostructures by clarifying ferroelectric polarizations and observing the improvement of the electronic conduction at the phase boundary. Our findings provide new pathways to one-dimensional rewritable nanostructures and inspire researchers to conceive various multifunctional devices by combining the superb electromechanical property with their unique interfacial electronic conduction properties at nanoscale phase boundaries.

NPG Asia Materials (2014) 6, e81; doi:10.1038/am.2013.72; published online 17 January 2014

Keywords: ferroelectrics; morphotropic phase boundary; multiferroics; nanostructure

\section{INTRODUCTION}

Morphotropic phase boundary systems have attracted a considerable amount of attention because of their electromechanical functionality. ${ }^{1}$ Large piezoelectric susceptibilities are observed at the morphotropic phase boundaries, which occur at the compositional phase boundary of tetragonal and rhombohedral structures in lead-based piezoelectric compounds. ${ }^{2-4}$ Such a morphotropic phase boundary, along with a vast improvement of piezoelectricity, can be induced in a simple tetragonal perovskite $\mathrm{PbTiO}_{3}$ under a high-pressure environment. ${ }^{5,6}$

Recently, a strain-driven morphotropic phase boundary was realized in $\mathrm{BiFeO}_{3}$ (BFO) thin films by the use of heteroepitaxial strain. ${ }^{7}$ The BFO has been actively studied as a multiferroic compound because it shows ferroelectricity $\left(T_{\mathrm{C}} \sim 1103 \mathrm{~K}\right)$ and antiferromagnetism $\left(T_{\mathrm{N}} \sim 640 \mathrm{~K}\right)$ at room temperature. ${ }^{8,9}$ The bulk phase of BFO has a rhombohedral structure (space group $R 3 c$ ), and its weakly strained epitaxial films show a ferroelectric polarization $\left(\sim 100 \mu \mathrm{C} \mathrm{cm}^{-2}\right)$ along the $<111>$ direction. ${ }^{10,11}$ Moreover, the growth of BFO on $(001) \mathrm{LaAlO}_{3}$ substrates $(a \sim 3.79 \AA)$ stabilizes a new highly elongated phase with large tetragonality $(c / a \sim 1.23)^{7,12}$ in which the structural, magnetic and ferroelectric properties are largely modified. ${ }^{13-18}$ The $T_{\mathrm{N}}$ greatly decreases to $\sim 370 \mathrm{~K}$ and ferroelectric reorientation transition accompanied with structural phase transition between $\mathrm{M}_{\mathrm{C}^{-}}$and $\mathrm{M}_{\mathrm{A}}$-type monoclinic structures occurs concomitantly at the $T_{\mathrm{N}} \cdot{ }^{13,15,18}$

With increasing thickness, the mixed-phase regions are formed and composed of the highly elongated phase (T-phase) and a third polymorphic phase with relatively smaller $c$-axis lattice parameter ( $\mathrm{R}^{\prime}$-phase). Because the relevant competing phases have quite different $c$-axis lattice parameters, as much as $10 \%$ while still being fully epitaxial without cracks or grain boundaries, electric control of the tetragonal-rhombohedral $\left(T-\mathrm{R}^{\prime}\right)$ phase boundary $\left(\mathrm{T}-\mathrm{R}^{\prime} \mathrm{PB}\right)$ holds promise for very large electromechanical responses. ${ }^{7,19}$ Particular attention has been paid to carefully unveil the exact crystal structures of these two constituent phases in the presence of complex mechanical deformation and strain gradient within the mixed-phase areas. Damodaran et al. ${ }^{20}$ have described the $\mathrm{R}^{\prime}$ - and T-phase as monoclinic structures, denoted by $\mathrm{M}_{\mathrm{I}}$ and $\mathrm{M}_{\mathrm{II}}$, respectively, and Chen et al. ${ }^{21}$ have introduced triclinic distortions to describe them.

Tailoring the mixed-phase regions is crucial to further understand the nature of phase competition and to realize the various electromechanical applications related to the exotic functionalities, such as

\footnotetext{
${ }^{1}$ Department of Physics, Korea Advanced Institute of Science and Technology (KAIST), Daejeon, Republic of Korea; ${ }^{2}$ School of Materials Science and Engineering, University of New South Wales, Sydney, New South Wales, Australia; ${ }^{3}$ Center for Nanomaterials and Chemical Reactions, Institute for Basic Science (IBS), Daejeon, Republic of Korea; ${ }^{4}$ Department of Materials Science and Engineering, KAIST, Daejeon, Republic of Korea and ${ }^{5}$ nstitute for the NanoCentury, KAIST, Daejeon, Republic of Korea

Correspondence: Professor C-H Yang, Department of Physics, Korea Advanced Institute of Science and Technology (KAIST), 291 Daehak-ro, Yuseong-gu, Daejeon 305-701, Republic of Korea.

E-mail: chyang@kaist.ac.kr
}

Received 8 July 2013; revised 6 October 2013; accepted 27 October 2013 
giant electrostrain and spontaneous magnetic moments observed in the mixed-phase regions. ${ }^{19,22}$ Numerous efforts have been made to control the $\mathrm{T}-\mathrm{R}^{\prime} \mathrm{PB}$ of $\mathrm{BFO}$ thin films through an external electric field, substrate vicinality and biaxial heteroepitaxial strain, ${ }^{23-27}$ but stripe $\mathrm{T}-\mathrm{R}^{\prime} \mathrm{PBs}$ with long-range continuity have not yet been obtained. Consequently, the shape of T- $\mathrm{R}^{\prime} \mathrm{PB}$ in pure BFO is not a straight line but a slightly curved one, ${ }^{7,24,28}$ making it hard to attain stripe mixed-phase patterns with a long-range spatial continuity.

In this study, we show that a La 5\% substitution into BFO thin films results in the formation of well-aligned straight stripe $\sim 100 \mathrm{~nm}$ wide $T-\mathrm{R}^{\prime} \mathrm{PBs}$, which changes their potential usability in nanodevices dramatically. In addition, the alignment of $T-\mathrm{R}^{\prime} \mathrm{PB}$ is controlled deterministically with excellent spatial continuity and reliability over tens of microns, which allows various patterns of ferroelectric domains and interfaces based on these long-range straight stripe phases. Moreover, the stripe mixed-phase regions show a considerable improvement in interfacial electronic conduction at the phase boundary, offering new possibilities for nanoscale functionality.

\section{MATERIALS AND METHODS}

Thin $\mathrm{La}(5 \%)$-substituted $\mathrm{BFO}$ thin films $\left(\mathrm{Bi}_{0.95} \mathrm{La}_{0.05} \mathrm{FeO}_{3}\right.$, $\left.\mathrm{BLFO}\right)$ were grown with a conducting buffer layer of $\mathrm{Pr}_{0.5} \mathrm{Ca}_{0.5} \mathrm{MnO}_{3}$ on (001) $\mathrm{LaAlO}_{3}$ substrates using pulsed laser deposition with a $\mathrm{KrF}$ (krypton fluoride laser) excimer laser $(\lambda=248 \mathrm{~nm})$. We used a BLFO ceramic target $\left(\mathrm{Bi}_{1.05} \mathrm{La}_{0.05} \mathrm{FeO}_{3}\right)$ with $\mathrm{Bi}$ at $10 \%$ excess to prevent bismuth loss during pulsed laser deposition growths. The laser energy was $110 \mathrm{~mJ}$, and the frequency was $10 \mathrm{~Hz}$. During growth, the substrate temperature was kept at $650{ }^{\circ} \mathrm{C}$ and oxygen partial pressure was set to 100 mTorr. After growth was completed, the samples were cooled to room temperature at a rate of $10^{\circ} \mathrm{C}$ per min with an oxygen environment of 500 Torr. The film thickness of the BLFO layer was $\sim 60 \mathrm{~nm}$. The surface topography and piezoelectric domain structure of BLFO were investigated by a scanning probe microscopy (Veeco multimode-V) using Ti/Pt-coated conductive tips (MikroMasch, Lady's Island, SC, USA) with a typical scan rate of $7-9 \mu \mathrm{m} \mathrm{s}^{-1}$. All the surface topographic images in the study were displayed so that the horizontal and vertical lines were parallel to the crystallographic [100] and [010] axes of $\mathrm{LaAlO}_{3}$ substrate, respectively. For the transmission electron microscopy (TEM) measurements, a cross-sectional TEM sample was prepared by a Focused Ion Beam (Quanta 3D FEG, FEI Company, Hillsboro, OR, USA) and then examined using JEOL JEM-3010 operated at $300 \mathrm{keV}$. Conductive atomic force microscope measurements were performed at room temperature using an AIST-NT Smart SPM 1000 and Ti-Pt-coated cantilevers. The crystal structure of the BLFO film was investigated using an X-ray diffractometer (PANalytical X'pert MRD Pro) with $\mathrm{Cu} \mathrm{K}_{\alpha 1}$ radiation.

\section{RESULTS AND DISCUSSION}

Figure 1a Shows a topographic image of a BLFO film in the as-grown state. The mixed-phase regions of BLFO films can be identified by the typical surface structure, where two competing phases appear alternately on a 100 nanometer length scale. Because of the smaller $c$-axis lattice parameter of the $\mathrm{R}^{\prime}$-phase, we can observe the mixedphase regions in the topographic image. In comparison with the mixed-phase regions of pure BFO, the mixed-phase regions of BLFO are made of straight $\mathrm{T}-\mathrm{R}^{\prime}$ PBs with a good spatial continuity. In pure $\mathrm{BFO}$, the $\mathrm{T}-\mathrm{R}^{\prime} \mathrm{PBs}$ are slightly bent and cut into segments, which would make it difficult to get uniform and continuous PBs. As presented in Figure 1b, this characteristic of a straight-line shape results in a more dramatic appearance of stripe $\mathrm{T}-\mathrm{R}^{\prime} \mathrm{PBs}$ in electrically formed regions.

We applied an electric field to the sample while scanning a $5 \times 5 \mu \mathrm{m}$ square area using a conductive tip with a negative voltage of $-10 \mathrm{~V}$. Consequently, the out-of-plane polarization, although it is not presented here, is switched from down to up. Simultaneously, we can get the formation of straight stripe $\mathrm{T}-\mathrm{R}^{\prime} \mathrm{PBs}$, which aligns along two orientations tilted by $+12^{\circ}$ or $-12^{\circ}$ with respect to the crystalline axis [010]. As in pure BFO, the PB alignments prefer one of four axes, which are tilted with respect to the crystalline axes [100] or [010] in morphological aspects. ${ }^{28}$ It has been reported that each $\mathrm{PB}$ line in pure BFO can have two additional subvariants with different net polarization directions. ${ }^{24}$ The selection of the two morphological orientations is related to the tip scanning direction. Details of the effect of the tip scanning direction on the alignment will be discussed later.

In contrast, we have observed that additional poling with a positive voltage of $+10 \mathrm{~V}$ on the tip can erase all the T- $\mathrm{R}^{\prime}$ PBs cleanly. A similar erasing process has been demonstrated in pure BFO films by Zeches et al. ${ }^{7}$ As presented in Figure $1 \mathrm{c}$, the positively poled square area shows an atomically flat surface of pure T-phase, even revealing a step terrace structure. Therefore, we can write or erase stripe T-R' PBs by applying negative or positive $d c$ voltage to the tip. Notably, the different top/bottom boundary conditions may drive an imprint, preferring an out-of-plane polarization direction; in this case, we observed that the as-grown state has the polarization pointing down. Regardless of the same external voltage magnitude, it is likely that the effective electric field the BLFO layer experiences in the erasing process is stronger because of the additional built-in field than the writing process. The different behavior depending on the polarity of bias voltage can be understood in the context of an asymmetric imprint in the hysteresis curve of strain versus electric field. ${ }^{7}$

To clarify the nanoscale crystalline structure of mixed-phase region in BLFO, we carried out TEM analysis for a cross-sectional area of a BLFO film. A TEM specimen was prepared with an approximate 50$\mathrm{nm}$ thickness along the zone axis of [010] to keep the mixed-phase region in place from strain relaxation. The cross-sectional bright and dark field images exhibit a typical mixed-phase structure with a
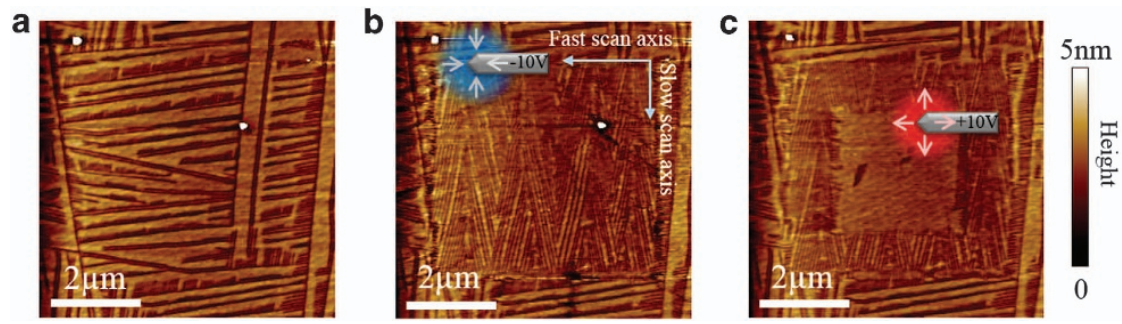

Figure 1 (a) A topographic image for the as-grown surface of a BLFO film. (b) A topographic image for the area poled by a scanning tip with a negative bias of $-10 \mathrm{~V}$. Two-variant stripe T-R' PBs are produced as a result of the poling with the slow scan along [010] crystalline axis. (c) The written stripe mixedphase areas can be erased by a poling with a positively biased $(+10 \mathrm{~V})$ tip. The poled area is transformed to pure tetragonal-like phase with step terrace structure. The schematics of tips represent the scan direction and the bias voltage. 

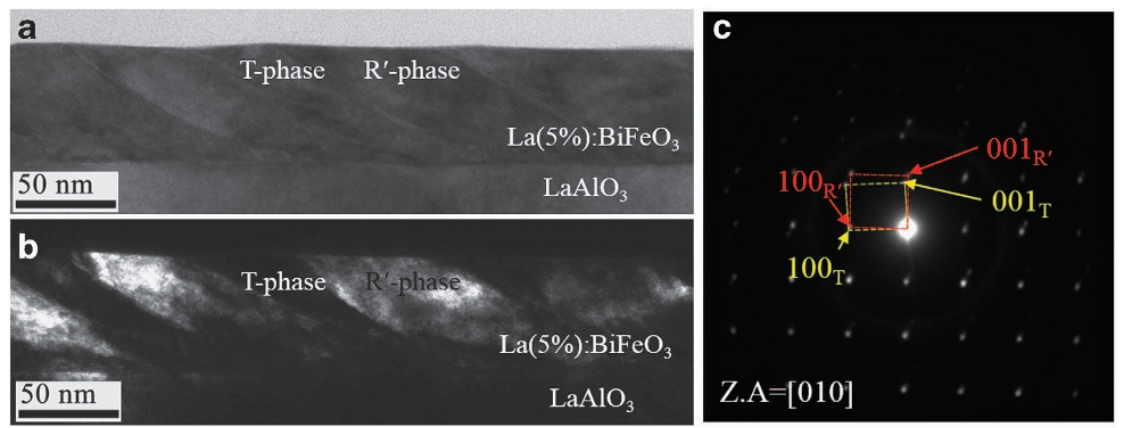

Figure 2 (a) Bright field TEM image for the cross-sectional area of mixed phase region in a BLFO thin film on (001) LAO substrate. (b) Dark field TEM micrograph acquired from the (001) diffraction spot of $R^{\prime}$-phase using objective aperture. (c) Selected area electron diffraction (SAED) pattern of the BLFO layer. Yellow (red)-colored dashed lines represent the reciprocal pseudo-cubic unit cell of T-phase ( $\mathrm{R}^{\prime}$-phase).
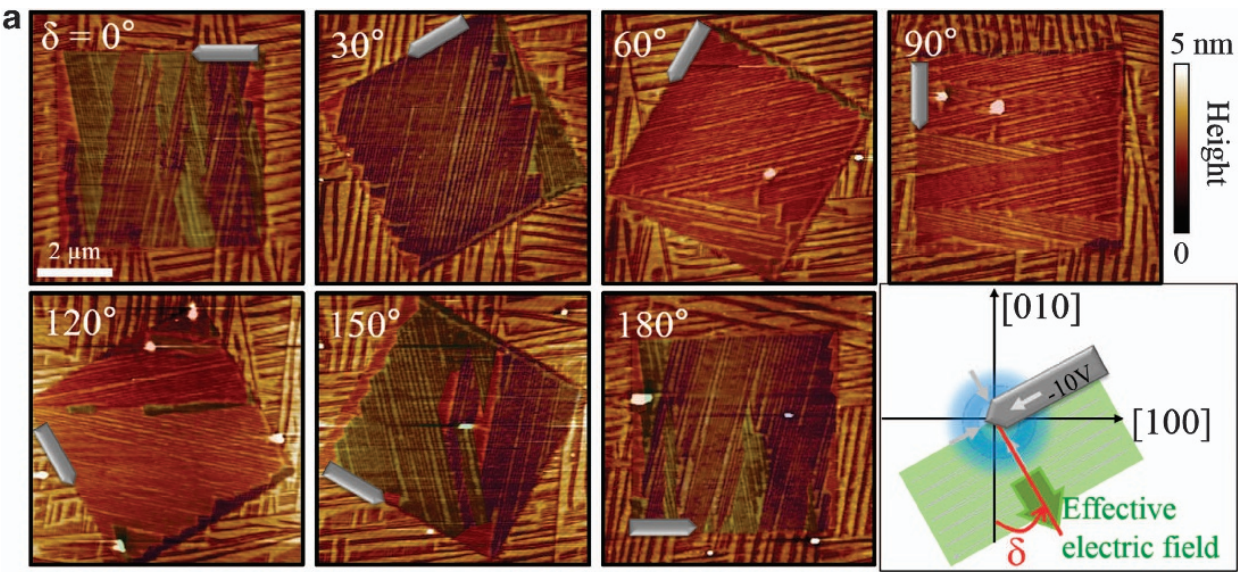

b

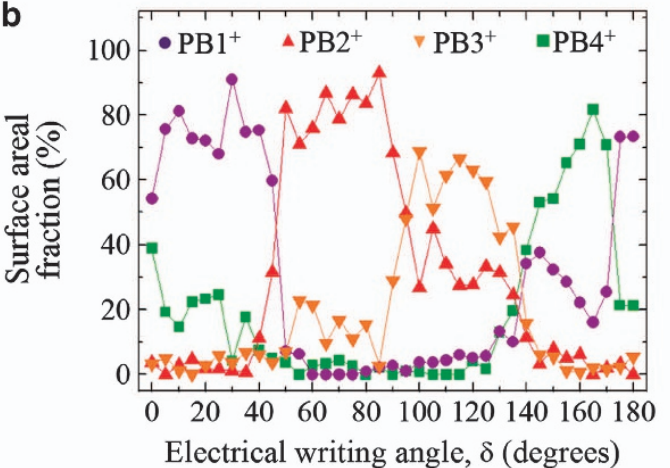

C

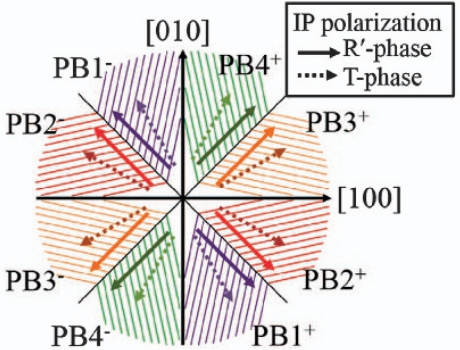

Figure 3 (a) Topographic images of electrically formed T-R' PBs obtained varying the electrical writing angle ( $\delta$ ). There exist four-variant stripe PB regions and each region is overlaid with different colors (purple, red, orange and green). Schematics of tip indicate the fast scan directions during the electrical poling with $-10 \mathrm{~V}$. Each image size is $7 \times 7 \mu \mathrm{m}$. The electrical writing angle $(\delta)$ is defined to be an inter-angle between a slow scan direction and a crystalline axis of [0-10]. (b) Surface areal fractions of each mixed-phase region within the electrically written square box are presented as a function of the electrical writing angle. (c) The colors distinguish the aligning axes of four-variant stripe T-R' PBs.

recurring structure consisting of T-phase and $\mathrm{R}^{\prime}$-phase (see Figures 2a and $\mathrm{b}$ ). The typical saw-tooth shaped surface termination is barely detectable on the top, which provides insight on the surface morphology of mixed-phase region. Figure $2 \mathrm{c}$ shows a selected-area electron diffraction pattern on the BLFO layer. Because the dark field TEM micrograph is acquired from the $(001) \mathrm{R}^{\prime}$-phase peak, bright regions in the dark field image indicate the $\mathrm{R}^{\prime}$-phase region. The pseudo-cubic unit cells of $\mathrm{T}$ - and $\mathrm{R}^{\prime}$-phases are tilted against each other by $\sim 4$ degrees around the zone axis of [010]. The lattice parameters are $a \sim 3.7(5) \AA$ and $c \sim 4.7(5) \AA$ for the T-phase and $a \sim 3.8 \AA$ and $c \sim 4.2 \AA$ for the $\mathrm{R}^{\prime}$-phase (see Supplementary Figure S1 for high-resolution TEM image). From the cross-sectional TEM study, we know that the internal mixed-phase structure of BLFO is qualitatively similar to that of pure BFO, despite the fairly different stripe feature on the topographic images. Notably, the phase boundaries are inclined with respect to the substrate surface on the top surface up to $\sim 33^{\circ}$ and $23^{\circ}$ for the $T \backslash \mathrm{R}^{\prime}$-type (connected to the maximum height position on surface) and $\mathrm{R}^{\prime} \backslash \mathrm{T}$-type (to the 
minimum height) boundaries, respectively. However, the boundaries are curved and become more parallel to the substrate surface near the interface between the film and substrate.

To investigate whether the alignment of stripe T-R $\mathrm{R}^{\prime} \mathrm{PBs}$ can be controlled by the tip scan direction in writing process, we measured topographic images over the written areas varying the slow scan direction, which is rotated counterclockwise from [0-10] at an interval of $5^{\circ}$. In Figure $3 \mathrm{a}$, we plotted the representative resultant images and overlaid semi-transparent color plates on the corresponding mixed-phase regions to gain a clear of the different T-R' PBs (See Supplementary Figure S2 for full set of measured data). We observed that the predominant $\mathrm{T}-\mathrm{R}^{\prime} \mathrm{PB}$ alignments strongly depend on the fast scan direction, although other competing alignments appeared, especially at the side edges. The areal fractions of the four competing mixed-phase regions are quantitatively estimated and presented as functions of the fast scan direction in Figure 3b. The schematic of Figure $3 c$ describes color maps to depict the four-variant $T-R^{\prime} P B$ axes. The writing angle space is divided into eight sectors each of that possesses 45 degrees in the interval, respectively. In fact, each T-R' PB axis has two additional variants due to ferroelectric polarization.

To clarify the in-plane electric polarization of the T- and $\mathrm{R}^{\prime}$-phase within written mixed-phase areas of BLFO, we performed lateral piezoresponse force microscopy (PFM) by rotating the direction of cantilever azimuthally. The lateral PFM can sensitively detect the inplane component perpendicular to the cantilever by the use of the tip torsional mode. We made two neighboring $\mathrm{T}^{-} \mathrm{R}^{\prime} \mathrm{PB}_{2}{ }^{+}$and $\mathrm{PB} 2^{-}$ regions by electrical writings of $\delta=60^{\circ}$ and $240^{\circ}$ for efficiently checking the $180^{\circ}$ rotational symmetry. Surface topography and lateral PFM are simultaneously measured using a tip direction along [ -100$]$ and plotted in Figure 4a. It can be clearly seen that the stripe mixed-phase areas consist of wide T-phase areas and relatively narrow $\mathrm{R}^{\prime}$-phase regions. Because the PFM contrast corresponds to the real part of the $a c$ response of the tip torsional vibration, the $\mathrm{PB} 2{ }^{+}$and $\mathrm{PB} 2^{-}$areas should have similar magnitude but different sign in phase on the corresponding positions, which are exactly identified in the measured result. Varying the tip orientation direction, we find the azimuthal angles at which the sign of the real part of PFM signal changes. The resultant images are plotted in Figure $4 \mathrm{~b}$ and the representative PFM signals for $\mathrm{T}$ - and $\mathrm{R}^{\prime}$ - phase are extracted from the same areas marked by red (or blue) spots in each panel. Figure $4 \mathrm{c}$ summarizes the PFM signal as a function of the tip orientation angle. The sign of PFM signal changes at $30( \pm 3)^{\circ}$ for T-phase and $47( \pm 3)^{\circ}$ for $\mathrm{R}^{\prime}$-phase, which indicate that each in-plane polarization direction is along the axes. Figure $4 \mathrm{~d}$ shows a schematic of in-plane polarization directions of the T-phase and $\mathrm{R}^{\prime}$-phase in $\mathrm{PB} 2^{+}$and $\mathrm{PB} 2^{-}$regions. The surface area of T-phase is $\sim 1.5$ times wider than $\mathrm{R}^{\prime}$-phase but the magnitude of polarization for T-phase is $\sim 1.3$ times smaller than that for the $\mathrm{R}^{\prime}$-phase. Therefore, we estimate that the net in-plane polarization is tilted by $\sim 38^{\circ}$ with respect to the crystal axis of [100]. In addition, the out-of-plane PFM image for the identical region suggests that the $\mathrm{T}$ - and $\mathrm{R}^{\prime}$-phase areas have relatively uniform out-of-plane PFM signals (See Supplementary Figure S3). This is a different feature from the pure BFO, in which the out-of-plane PFM shows a strong signal at the phase boundaries rather than the

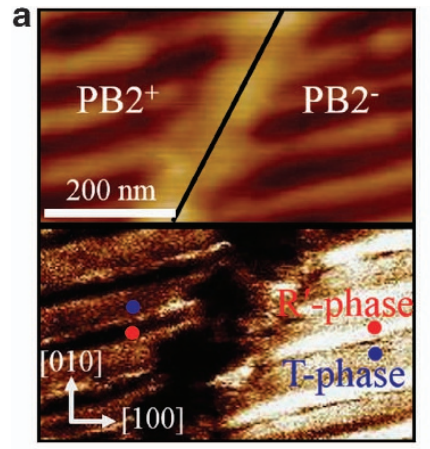

c

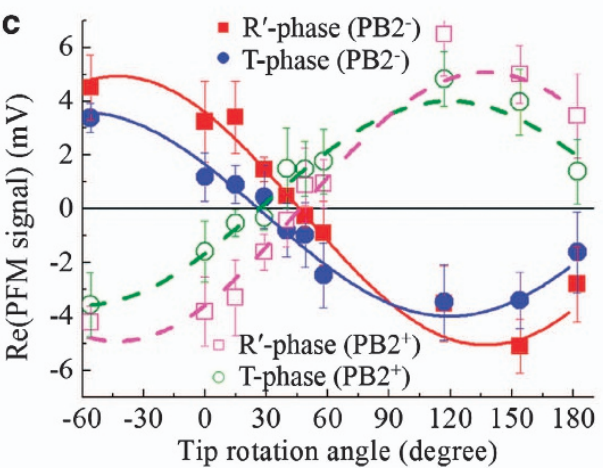

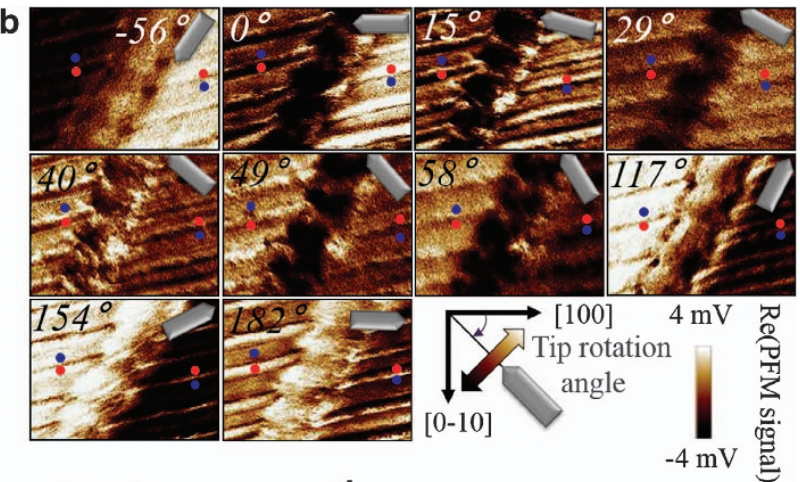

d

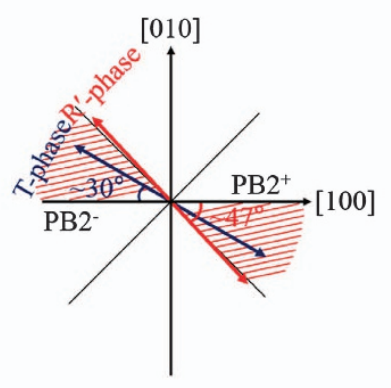

Figure 4 Angle-resolved lateral PFM measurements define in-plane polarization directions in the mixed-phase regions. (a) Topographic and lateral PFM images. Red and blue dots indicate the representative locations of $\mathrm{R}^{\prime}$-phase and T-phase, respectively. (b) Lateral PFM images on the same area were measured, changing tip orientation angle as shown in an illustration of a tip using an ac tip voltage of $3 \mathrm{~V}$. Inside each image, red and blue dots indicate the same positions as those in (a), which are used for comparisons and data extractions. (c) Lateral PFM signals of the T-phase and R'-phase for PB2 ${ }^{+}$and $\mathrm{PB}^{-}$- regions are quantitatively extracted from the images in (b) and plotted as functions of tip orientation angle. The lateral PFM signal is proportional to the in-plane polarization component projected to the perpendicular axis of tip; thus, the signals show sinusoidal dependence on the tip orientation. Solid (dashed) curves for $\mathrm{PB}^{-}-\left(\mathrm{PB} 2^{+}\right.$) regions are obtained by fitting the data to sinusoidal functions with putting phase shift and amplitude as variables. (d) A schematic shows the measured in-plane polarization directions of T-BFO and $\mathrm{R}^{\prime}-\mathrm{BFO}$ phases in the PB2 ${ }^{+}$and PB2 ${ }^{-}$regions. 

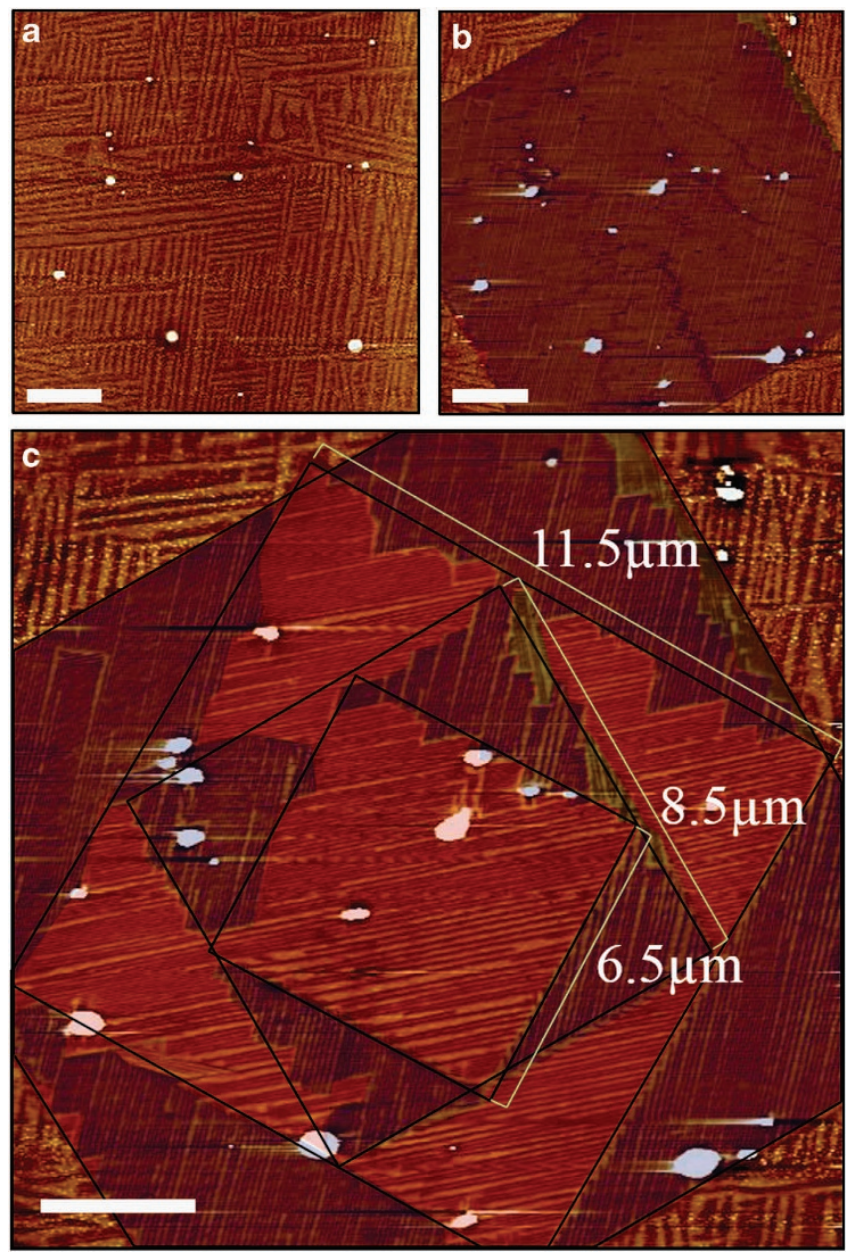

Figure 5 (a) Surface morphology of the as-grown state of BLFO. (b) A surface morphology image measured after an electrical writing along $\delta=30^{\circ}$ on a $16 \times 16 \mu \mathrm{m}$ square box. (c) Surface morphology for the region where three more overwriting processes were performed with different writing angles sequentially: $\delta=60^{\circ}$ for the $11.5 \mu \mathrm{m}$ square box, $\delta=30^{\circ}$ for the $8.5 \mu \mathrm{m}$ box and $\delta=60^{\circ}$ for the $6.5 \mu \mathrm{m}$ box. All writing processes were conducted using a negatively biased $(-10 \mathrm{~V})$ tip. Scale bars represent $3 \mu \mathrm{m}$.

constituent phase areas. ${ }^{29}$ The $\mathrm{R}^{\prime}$-phase region exhibits an $\sim 15 \%$ higher out-of-plane piezoresponse than the T-phase region. With the assumption that the piezoresponse is proportional to ferroelectric polarization, it is presumed that the ferroelectric polarization of T-phase $\left(\vec{P}_{T}\right)$ within the mixed-phase area has a magnitude of $\sim 120 \mu \mathrm{Ccm}^{-2}$ and orients itself to a direction rotated by a polar angle of $15^{\circ}$ from the normal and by an azimuthal angle of $30^{\circ}$ from an in-plane direction $<100>$. The polarization of $\mathrm{R}^{\prime}$-phase $\left(\vec{P}_{R^{\prime}}\right)$ is $\sim 140 \mu \mathrm{C} \mathrm{cm}^{-2}$ and its direction is represented by a polar angle of $18^{\circ}$ and an azimuthal angle of $47^{\circ}$.

The tip-based manipulation of ferroelectric domain walls has been explored in many studies. ${ }^{23,24,30-32}$ Electric fields formed by applying a negative voltage to the tip are composed of in-plane components converging inward at the tip as well as an out-of-plane directional field component. During tip scanning, the electric fields along the fast scan axis countervail each other; even the in-plane electric field antiparallel to the slow scan direction does not have the final influence on the mixed-phase formation because the written effect
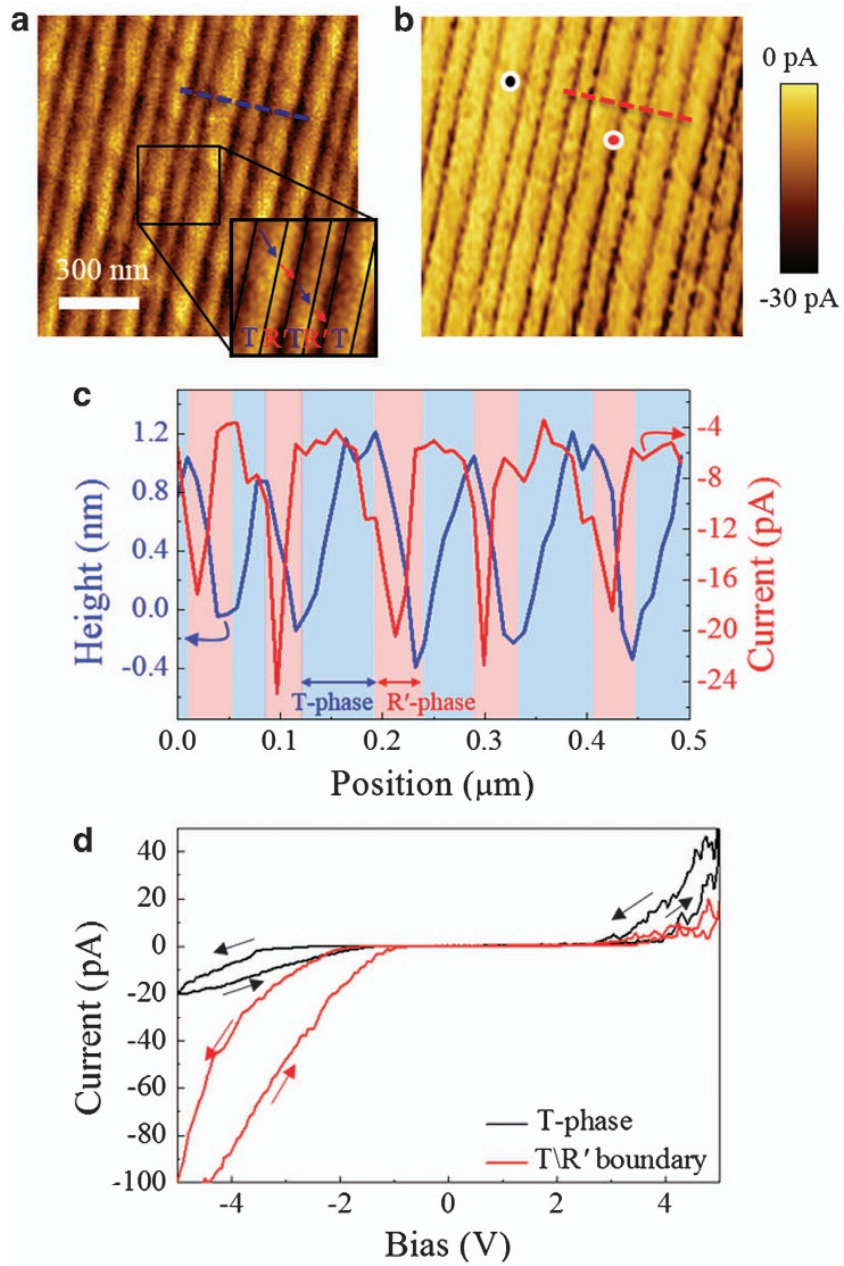

Figure 6 Conductive atomic force microscope (AFM) characterization of $\mathrm{T}-\mathrm{R}^{\prime}$ phase boundaries. (a) AFM-topography for a written $\mathrm{PB} 1^{+}$region; the inset displays the corresponding in-plane polarizations. (b) Simultaneous conductive-AFM current image measured at $-4.5 \mathrm{~V}$ bias. Red and black points show representative positions for I-V data shown in (d). (c) Current and topography cross sections showing current maxima location at the $T-\mathrm{R}^{\prime}$ phase boundaries taken at positions of red and blue lines in (a) and (b). (d) I-V curves at high current ( $T \backslash \mathrm{R}^{\prime}$ boundaries) and low current areas (T-phase) taken at representative positions shown in (b).

is erased and reversed by the opposite field in the next several line scans. Notably, the scans with a negative voltage applied to the tip give rise to effective in-plane fields toward the slow scan direction. Accordingly, the alignment of $\mathrm{T}-\mathrm{R}^{\prime} \mathrm{PBs}$ can be understood by the interaction between the tip-induced effective electric field and a certain net in-plane polarization of the mixed-phase area. You et al. ${ }^{24}$ have clarified the net in-plane polarization direction using angleresolved PFM for pure BFO; the alignments of T- $\mathrm{R}^{\prime} \mathrm{PBs}$ are opposite to the effective electric field direction at the rear side of the slow scan direction, possibly because of the elastic constraint imposed by the formed mixed phases. However, the stripe mixed phases of BLFO is determined by the net polarization orienting itself to the effective electric field. This is another distinct point for BLFO when compared with BFO. The discrepancy is thought to be a consequence of the relative absence of a T-phase matrix in the writing process of BLFO owing to the long-range continuity. Notably, the edge regions of poled 

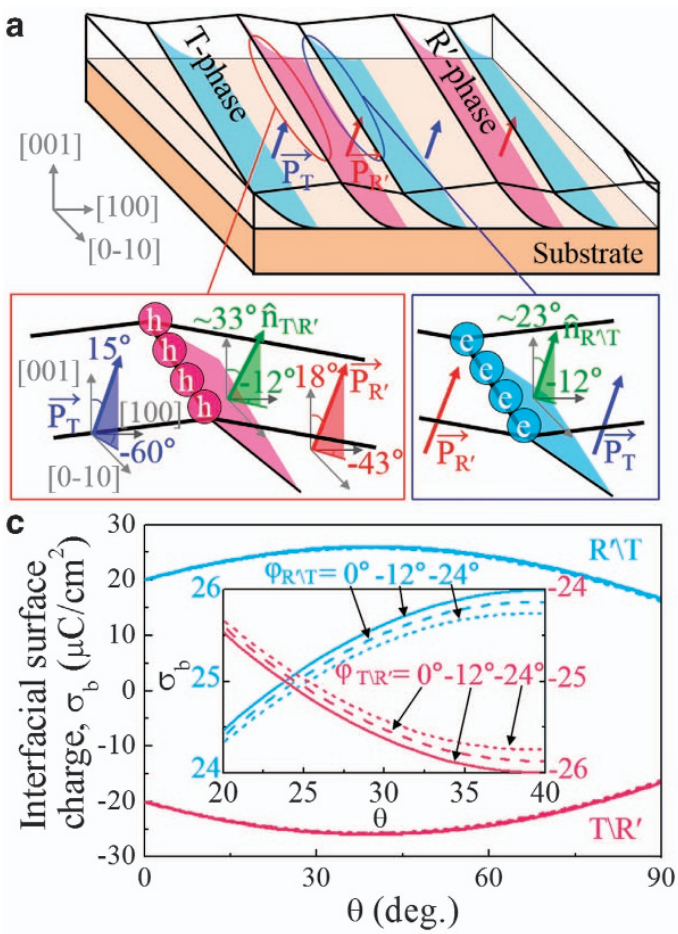
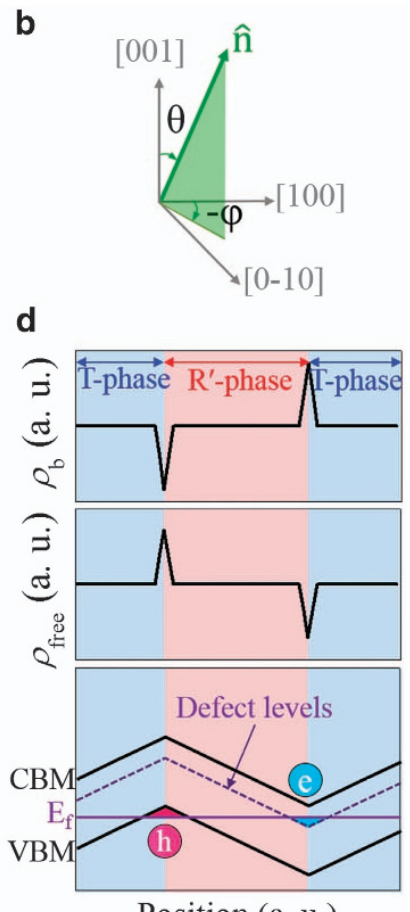

Position (a. u.)

Figure 7 A proposed scenario of electronic conduction. (a) A schematic of PB1 ${ }^{+}$-type mixed-phase area. Polarization discontinuity at the phase boundaries produce charged domain walls where hole or electron carriers are collected for screening the bound charges. (b) The normal vector of interfacial plane: its direction is indicated by the polar angle $(\theta)$ and azimuthal angle $(\varphi)$. (c) The surface bound charge densities $\left(\sigma_{b}\right)$ at the interfaces are plotted as a function of $\theta$ at selected values of $\varphi$. (d) The bound charge is due to ionic charges, and free electronic charges are collected to screen partially the bound charges. The net total charges near the interfaces produce a built-in field in between, thereby providing band bending and electron/hole carriers at the interfaces in a self-consistent way. The $n$-type carriers are trapped in the shallow mid-gap states arising from the impurities.

square boxes undergo effective in-plane electric fields orienting to the inward-pointing normal of edges, which are perpendicular to the slow scan direction prevailing far inside the poling square boxes. The observed difference in the $\mathrm{T}-\mathrm{R}^{\prime} \mathrm{PB}$ formation at the edges can be attributed to the special feature of the tip-induced field in the edge regions. With this in mind, we infer that large area forming can be helpful to minimize the edge effect and obtain a better single variant mixed-phase area.

We attempted to form stripe $\mathrm{T}-\mathrm{R}^{\prime} \mathrm{PBs}$ on a larger area of a $16 \times 16 \mu \mathrm{m}$ square box. As shown in Figures $5 \mathrm{a}$ and $\mathrm{b}$, the area can be filled with almost entirely single variant stripe mixed-phase area (type of $\mathrm{PB}^{+}$) by a tip-based electrical forming with $\delta=30^{\circ}$. It took $\sim 15 \mathrm{~min}$ to form the area using a tip sweeping speed of $9 \mu \mathrm{m} \mathrm{s}^{-1}$ and 256 lines. The typical formed stripe T-R $\mathrm{R}^{\prime}$ PBs are as dense as $90 \mathrm{~nm}$ apart from each other. Some stripe PB lines have good spatial continuity to such an extent that they are extended to the opposite side of the poling box. In principle, if one could scan the corresponding sizable area, there is no limitation in the length of stripe PB lines. As previously stated, it is feasible to erase the written T- $\mathrm{R}^{\prime}$ PBs by poling with a positive bias. Moreover, we show that the written area can be overwritten to the other directional mixed-phase areas. By changing only the sweeping direction from $\delta=30^{\circ}$ to $\delta=60^{\circ}$ without reversing the polarity of tip voltage, we can induce the formation of $\mathrm{PB}_{2}{ }^{+}$type (near-horizontal) alignment. We note that switching the out-of-plane polarization is not necessary to align PBs. We carried out additional electrical writing inside the box to make a type of $\mathrm{PB}^{+}$and a type of $\mathrm{PB}^{+}{ }^{+}$show up alternately. Figure $5 c$ exhibits the surface morphology over the area inside which three additional overwriting processes were conducted, sequentially decreasing the size of the poling box. In every process of overwriting, the stripe $\mathrm{T}-\mathrm{R}^{\prime} \mathrm{PB}$ was well aligned to the intended direction, according to the previous writing angle dependence. The experiment confirms that the character of the electric switching is reversible and nonvolatile.

Finally, we explore electronic conduction at a written stripe mixedphase area using conductive atomic force microscope. As presented in Figure 6, surface topography and local electronic conduction are measured simultaneously, which enables us to identify the exact spatial positions where electronic conduction is enhanced. The stripe mixed-phase area is of $\mathrm{PB}^{+}$type and in-plane polarizations orient to the directions as described in the inset of Figure 6a. Remarkably, we detected that local electronic conduction is enhanced whenever the conductive tip is located near the highest position in the topographic image, where the interfacial boundaries of the T-phase and $\mathrm{R}^{\prime}$-phase are located. ${ }^{22}$ The exact maximum current positions tend to be shifted slightly toward $\mathrm{R}^{\prime}$-phase regions possibly because the boundary location underneath is shifted toward the $\mathrm{R}^{\prime}$-phase region. We measured I-V curves with the tip fixed at the two representative positions of the $T$ - and $T \backslash R^{\prime}$ phase boundary at a voltage sweeping rate of $0.8 \mathrm{~V} \mathrm{~s}^{-1}$. This measurement clearly shows the electronic conduction in the $T \backslash R^{\prime}$-type boundary region has relatively enhanced. The I-V curves exhibit Schottky diode behavior with a forward bias at a negative tip voltage. The hysteretic behavior suggests that slow defect motion can be involved in the electronic conduction. These observations were obtained repeatedly at several different positions (See Supplementary Figure S4). 

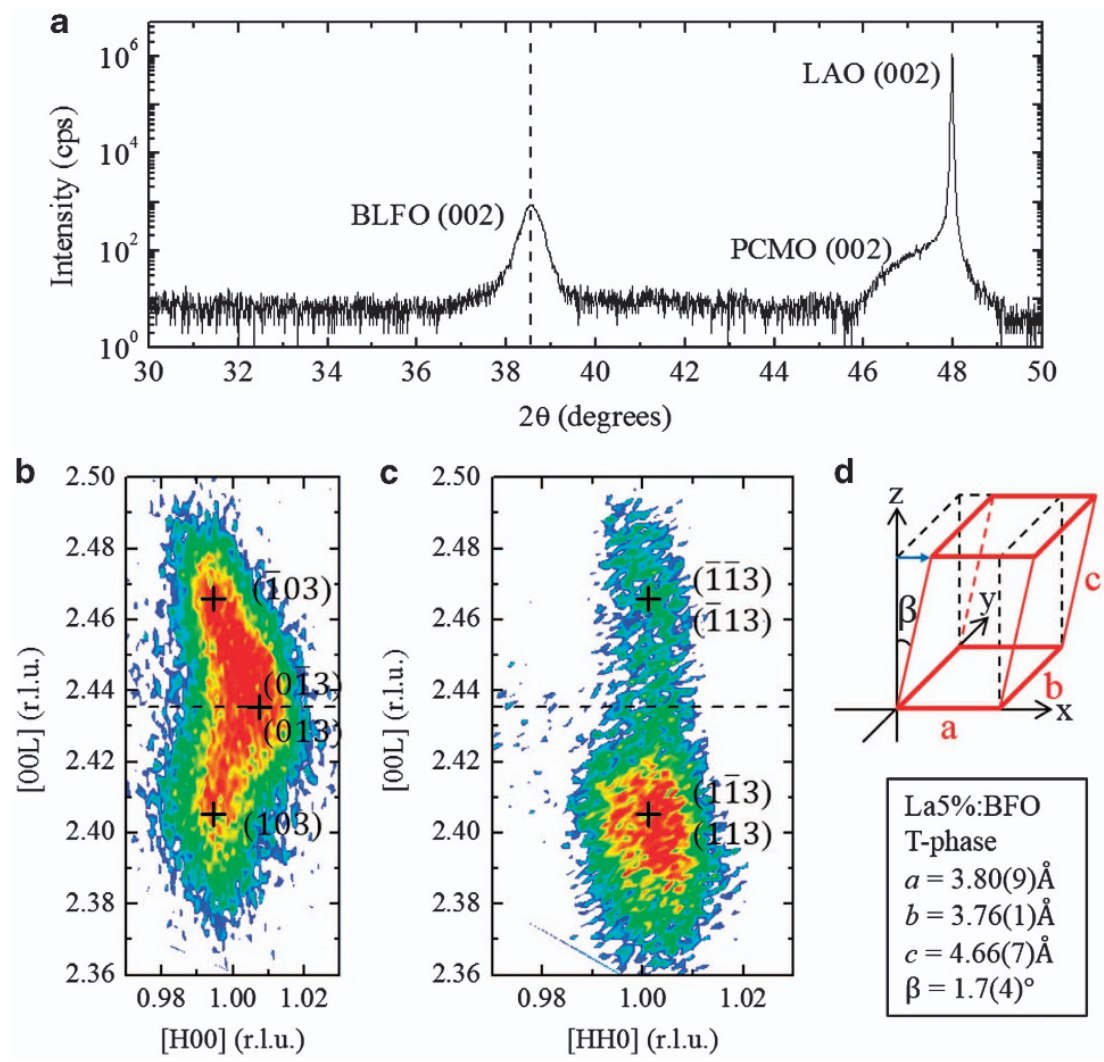

Figure 8 X-ray diffraction studies for a La 5\%-doped BFO thin film. (a) $\theta-2 \theta$ scan around LAO (002). Reciprocal space maps for (b) BLFO (103) and (c) BLFO (113) peaks. The reciprocal lattice unit (r.l.u.) is defined to be $2 \pi / 3.789 \AA^{-1}$, which corresponds to the reciprocal space value of the pseudo-cubic lattice parameter of LAO substrate. The horizontal dashed lines indicate the pseudotetragonal position expected from the (002) $\theta-2 \theta$ scan. (d) The crystal structure and lattice parameters determined from the reciprocal space maps.

To better understand why the electronic conduction is enhanced near the $T \backslash \mathrm{R}^{\prime}$-type boundaries located at the maxima, we consider the interfacial bound charge arising from the discontinuity of electric polarization at the boundaries and the relevant electronic charge accumulation. Given the ferroelectric polarizations of T-phase and R'phase, we can estimate the surface bound charge density $\left(\sigma_{b}\right)$ using relations of $\sigma_{b}=\left(\vec{P}_{T}-\vec{P}_{R^{\prime}}\right) \cdot \hat{n}_{T \backslash R^{\prime}}$ at the $\mathrm{T} \backslash \mathrm{R}^{\prime}$-type boundary and $\sigma_{b}=\left(\vec{P}_{R^{\prime}}-\vec{P}_{T}\right) \cdot \hat{n}_{R^{\prime} \backslash T}$ at the $\mathrm{R}^{\prime} \backslash \mathrm{T}$-type boundary, where $\hat{n}_{T \backslash R^{\prime}}$ and $\hat{n}_{R^{\prime} \backslash T}$ stand for the normal vectors of the corresponding boundaries (see Figure 7). As shown in the previous TEM image, the interfacial direction is modified at a different depth and also depends on the types of boundary. It was necessary to investigate $\sigma_{\mathrm{b}}$ as a function of the polar angle $(\theta)$ and azimuthal angle $(\varphi)$ of $\hat{n}$. Indeed, $\sigma_{\mathrm{b}}$ is insensitive to the variation of normal vectors, that is, the $\sigma_{\mathrm{b}}$ changes by less than $5 \%$ even if $\pm 10^{\circ}$ offset is introduced away from the given $\theta$ and $\varphi$ of $\hat{n}$. The bound charges at the interfacial region are partially screened by free electronic charges and the remaining unscreened charges (total ionic and electronic charges) produce a built-in field in between, leading to band bending. Accordingly, we can presume that type of carriers is holes at the maxima; however, we cannot observe any electronic conduction enhancement at the other boundary near the lowest height which is where electron carriers are expected to appear. It can be understood by introducing the presence of defects such as oxygen vacancies in the interfacial region where $n$-type carriers are pinned to the mid-gap states. In a previous study, we found that $n$-type-doped area of BFO by control of oxygen vacancies reveal prominently lower electronic conduction when compared with p-type doping areas. ${ }^{33}$

It is likely that $\mathrm{La}^{3+}$ ions substitute $\mathrm{Bi}^{3+}$ ions (A-site doping) because they have similar effective ionic radii $(1.36 \AA) .{ }^{34}$ Although the La substitutions would modify structural and ferroelectric properties such as domain size, coercive voltage and fatigue behavior, ${ }^{35,36}$ in many cases only the La 5\% substitutions do not cause dramatic changes in physical properties. However, due to the mechanically susceptible nature of morphotropic phase boundaries, such a small doping has a great impact on the formation of T-R' PBs. We carried out reciprocal space maps using a high-resolution X-ray four circle diffractometer to determine the crystalline structure of the BLFO film. As shown in Figure 8, the T-phase matrix of the BLFO film still has $\mathrm{M}_{\mathrm{C}}$-type splits of the (103) and (113) peaks, similar to those of pure BFO. The lattice parameters of monoclinic pseudo-cubic unit cell are $a_{\mathrm{m}} \sim 3.80(9) \AA, \quad b_{\mathrm{m}} \sim 3.76(1) \AA, \quad c_{\mathrm{m}} \sim 4.66(7) \AA$ and $\beta \sim 88.3^{\circ}$. The anisotropy of in-plane lattice parameters, $\left(a_{\mathrm{m}}-b_{\mathrm{m}}\right) / a_{\mathrm{m}}$, is reduced to $\sim 1.3 \%$ by the La $5 \%$ doping in comparison with $\sim 2.1 \%$ of pure BFO $\left(a_{\mathrm{m}} \sim 3.81 \AA, b_{\mathrm{m}} \sim 3.73 \AA, c_{\mathrm{m}} \sim 4.67 \AA, \beta \sim 88.2^{\circ}\right) .{ }^{15}$ A similar decreasing tendency by rare-earth doping has been reported in the $\mathrm{Sm}$ $10 \%$-doped BFO sample, which exhibited a much smaller anisotropy value of $\sim 0.5 \%{ }^{37}$ The origin of straight stripe $T-R^{\prime} P B$ is yet to be clearly understood. We encourage more rigorous theoretical and experimental studies on the possibility that the reduced anisotropy of in-plane lattice parameters offers better compatible matching between the $\mathrm{T}$ - and $\mathrm{R}^{\prime}$-phase within mixed-phase areas. The technique to form 
stripe PBs based on BLFO thin films might be exploited to yield various thermodynamically unstable domain structures at the boundary where two different $\mathrm{T}-\mathrm{R}^{\prime} \mathrm{PB}$ alignments collide. It will also be of great interest to further investigate new possibilities for the presence of exotic low-dimensional electronic conduction. Because the mixed-phase area is likely to have its peculiar ferroelectric and magnetic orders, which are possibly tied to the anisotropic crystalline structure, the present study may contribute to new conceptual electromechanical devices combined with electronic, magnetic and ferroelectric properties.

In summary, we reported on electrical formation and the control of long straight stripe T-R' PBs on BLFO thin films. Using electric fields generated from a $d c$ biased atomic force microscope tip, we can create, manipulate and erase the $\mathrm{T}-\mathrm{R}^{\prime} \mathrm{PB}$ with long-range spatial continuity in a rewritable way. The straight stripe $\mathrm{PBs}$ have advantages in getting a larger spatial area of uniform mixed-phases regions without leaving the T-phase matrix area. In addition, we observed that the interfacial boundary of the T-phase and $\mathrm{R}^{\prime}$-phase in BLFO showed a large improvement in electronic conduction possibly because of induced carriers arising from the polarization discontinuity. Our findings provide useful pathways into a variety of specific patterns of domains and interfacial boundaries such as charged domain walls and flux closure structures for future fundamental studies.

\section{CONFLICT OF INTEREST}

The authors declare no conflict of interest.

\section{ACKNOWLEDGEMENTS}

This work was supported by the National Research Foundation of Korea funded by the Ministry of Education, Science, and Technology (contract no. 2011-0016133). MJ and JYL were supported by Institute for Basic Science (IBS) [CA1301-03]. JS acknowledges support by the Australian Research Council (ARC) through a Future Fellowship (FT110100523). Work at KAIST was partially supported by the High Risk High Return Project. C-HY acknowledges support from the TJ Park Science Fellowship.

1 Noheda, B. \& Cox, D. E. Bridging phases at the morphotropic boundaries of lead oxide solid solutions. Phase Transit. 79, 5-20 (2006)

2 Jaffe, B., Roth, R. S. \& Marzullo, S. Piezoelectric properties of lead zirconate-lead titanate solid-solution ceramics. J. Appl. Phys. 25, 809-810 (1954).

3 Park, S.-E. \& Shrout, T. R. Ultrahigh strain and piezoelectric behavior in relaxor based ferroelectric single crystals. J. Appl. Phys. 82, 1804-1811 (1997)

4 Dkhil, B., Kiat, J. M., Calvarin, G., Baldinozzi, G., Vakhrushev, S. B. \& Suard, E. Local and long range polar order in the relaxor-ferroelectric compounds $\mathrm{PbMg}_{1 / 3} \mathrm{Nb}_{2 / 3} \mathrm{O}_{3}$ and $\mathrm{PbMg}_{0.3} \mathrm{Nb}_{0.6} \mathrm{Ti}_{0.1} \mathrm{O}_{3}$. Phys. Rev. B 65, 024104 (2001).

5 Ahart, M., Somayazulu, M., Cohen, R. E., Ganesh, P., Dera, P., Mao, H.-k., Hemley, R. J., Ren, Y., Liermann, P. \& Wu, Z. Origin of morphotropic phase boundaries in ferroelectrics. Nature 451, 545-548 (2008).

6 Wu, Z. \& Cohen, R. E. Pressure-induced anomalous phase transitions and colossal enhancement of piezoelectricity in $\mathrm{PbTiO}_{3}$. Phys. Rev. Lett. 95, 037601 (2005)

7 Zeches, R. J., Rossell, M. D., Zhang, J. X., Hatt, A. J., He, Q., Yang, C.-H., Kumar, A. Wang, C. H., Melville, A., Adamo, C., Sheng, G., Chu, Y.-H., Ihlefeld, J. F., Erni, R. Ederer, C., Gopalan, V., Chen, L. Q., Schlom, D. G., Spaldin, N. A., Martin, L. W. \& Ramesh, R. A strain-driven morphotropic phase boundary in $\mathrm{BiFeO}_{3}$. Science 326 , 977-980 (2009).

8 Roginskaya, Y. E., Tomashpol'skiï, Y. Y., Venevtsev, Y. N., Petrov, V. M. \& Zhdanov, G. S. The nature of the dielectric and magnetic properties of $\mathrm{BiFeO}_{3}$. Sov. Phys. JETP 23, 47-51 (1966).

9 Kiselev, S. V., Ozerov, R. P. \& Zhdanov, G. S. Detection of magnetic order in ferroelectric $\mathrm{BiFeO}_{3}$ by neutron diffraction. Sov. Phys. Dokl. 7, 742-744 (1963).

10 Lebeugle, D., Colson, D., Forget, A. \& Viret, M. Very large spontaneous electric polarization in $\mathrm{BiFeO}_{3}$ single crystals at room temperature and its evolution under cycling fields. Appl. Phys. Lett. 91, 022907 (2007).
11 Kim, D. H., Lee, H. N., Biegalski, M. D. \& Christen, H. M. Effect of epitaxial strain on ferroelectric polarization in multiferroic $\mathrm{BiFeO}_{3}$. Appl. Phys. Lett. 92, 012911 (2008)

12 Béa, H., Dupé, B., Fusil, S., Mattana, R., Jacquet, E., Warot-Fonrose, B., Wilhelm, F. Rogalev, A., Petit, S., Cros, V., Anane, A., Petroff, F., Bouzehouane, K., Geneste, G. Dkhil, B., Lisenkov, S., Ponomareva, I., Bellaiche, L., Bibes, M. \& Barthélémy, A. Evidence for room-temperature multiferroicity in a compound with a giant axial ratio. Phys. Rev. Lett. 102, 217603 (2009).

13 Ko, K.-T., Jung, M. H., He, Q., Lee, J. H., Woo, C. S., Chu, K., Seidel, J., Jeon, B.-G., Oh, Y. S., Kim, K. H., Liang, W.-I., Chen, H.-J., Chu, Y.-H., Jeong, Y. H., Ramesh, R., Park, J.-H. \& Yang, C.-H. Concurrent transition of ferroelectric and magnetic ordering near room temperature. Nat. Commun. 2, 567 (2011).

14 MacDougall, G. J., Christen, H. M., Siemons, W., Biegalski, M. D., Zarestky, J. L., Liang, S., Dagotto, E. \& Nagler, S. E. Antiferromagnetic transitions in tetragonal-like $\mathrm{BiFeO}_{3}$. Phys. Rev. B 85, 100406 (2012).

15 Chen, Z., Luo, Z., Huang, C., Qi, Y., Yang, P., You, L., Hu, C., Wu, T., Wang, J., Gao, C. Sritharan, T. \& Chen, L. Low-symmetry monoclinic phases and polarization rotation path mediated by epitaxial strain in multiferroic $\mathrm{BiFeO}_{3}$ thin films. Adv. Funct. Mater. 21, 133-138 (2011).

16 Kreisel, J., Jadhav, P., Chaix-Pluchery, O., Varela, M., Dix, N., Sánchez, F. \& Fontcuberta, J. A phase transition close to room temperature in $\mathrm{BiFeO}_{3}$ thin films. J. Phys.: Condens. Matter. 23, 342202 (2011).

17 Choi, K.-Y., Do, S. H., Lemmens, P., Wulferding, D., Woo, C. S., Lee, J. H., Chu, K. \& Yang, C.-H. Anomalous low-energy phonons in nearly tetragonal $\mathrm{BiFeO}_{3}$ thin films. Phys. Rev. B 84, 132408 (2011).

18 Siemons, W., Biegalski, M. D., Nam, J. H. \& Christen, H. M. Temperature-driven structural phase transition in tetragonal-like $\mathrm{BiFeO}_{3}$. Appl. Phys. Express. 4, 095801 (2011)

19 Zhang, J. X., Xiang, B., He, Q., Seidel, J., Zeches, R. J., Yu, P., Yang, S. Y., Wang, C. H., Chu, Y.-H., Martin, L. W., Minor, A. M. \& Ramesh, R. Large field-induced strains in a lead free piezoelectric material. Nat. Nanotechnol. 6, 98-102 (2011).

20 Damodaran, A. R., Liang, C.-W., He, Q., Peng, C.-Y., Chang, L., Chu, Y.-H. \& Martin, L. W. Nanoscale structure and mechanism for enhanced electromechanical response of highly strained $\mathrm{BiFeO}_{3}$ thin films. Adv. Mater. 23, 3170-3175 (2011).

21 Chen, Z., Prosandeev, S., Luo, Z. L., Ren, W., Qi, Y., Huang, C. W., You, L., Gao, C. Kornev, I. A., Wu, T., Wang, J., Yang, P., Sritharan, T., Bellaiche, L. \& Chen, L. Coexistence of ferroelectric triclinic phase in highly strained $\mathrm{BiFeO}_{3}$ films. Phys. Rev. $B$ 84, 094116 (2011).

22 He, Q., Chu, Y.-H., Heron, J. T., Yang, S. Y., Liang, W. I., Kuo, C. Y., Lin, H. J., Yu, P., Liang, C. W., Zeches, R. J., Kuo, W. C., Juang, J. Y., Chen, C. T., Arenholz, E., Scholl, A. \& Ramesh, R. Electrically controllable spontaneous magnetism in nanoscale mixed phase multiferroics. Nat. Commun. 2, 225 (2011)

23 Chen, Y.-C., He, Q., Chu, F.-N., Huang, Y.-C., Chen, J.-W., Liang, W.-I., Vasudevan, R. K. Nagarajan, V., Arenholz, E., Kalinin, S. V. \& Chu, Y.-H. Electric control of multiferroic orderings in mixed-phase $\mathrm{BiFeO}_{3}$ films. Adv. Mater. 24, 3070-3075 (2012).

24 You, L., Chen, Z., Zou, X., Ding, H., Chen, W., Chen, L., Yuan, G. \& Wang, J. Characterization and manipulation of mixed phase nanodomains in highly strained $\mathrm{BiFeO}_{3}$ thin films. ACS Nano 6, 5388-5394 (2012)

25 You, L., Yasui, S., Ehara, Y., Zou, X., Ding, H., Chen, Z., Chen, W., Chen, L., Funakubo, H. \& Wang, J. Domain tuning in mixed-phase $\mathrm{BiFeO}_{3}$ thin films using vicinal substrates. Appl. Phys. Lett. 100, 202901 (2012).

26 Zhang, J. X., Zeches, R. J., He, Q., Chu, Y.-H. \& Ramesh, R. Nanoscale phase boundaries: a new twist to novel functionalities. Nanoscale 4, 6196-6204 (2012).

27 Woo, C.-S., Lee, J. H., Chu, K., Jang, B.-K., Kim, Y.-B., Koo, T. Y., Yang, P., Qi, Y., Chen, Z., Chen, L., Choi, H. C., Shim, J. H. \& Yang, C.-H. Suppression of mixed-phase areas in highly elongated $\mathrm{BiFeO}_{3}$ thin films on $\mathrm{NdAlO}_{3}$ substrates. Phys. Rev. B 86, 054417 (2012)

28 Zhou, J., Trassin, M., He, Q., Tamura, N., Kunz, M., Cheng, C., Zhang, J., Liang, W.-I., Seidel, J., Hsin, C.-L. \& Wu, J. Directed assembly of nano-scale phase variants in highly strained $\mathrm{BiFeO}_{3}$ thin films. J. Appl. Phys. 112, 064102 (2012).

29 Mazumdar, D., Shelke, V., Iliev, M., Jesse, S., Kumar, A., Kalinin, S. V., Baddorf, A. P. \& Gupta, A. Nanoscale switching characteristics of nearly tetragonal $\mathrm{BiFeO}_{3}$ thin films. Nano Lett. 10, 2555-2561 (2010).

30 Ivry, Y., Chu, D. P., Scott, J. F. \& Durkan, C. Flux closure vortexlike domain structures in ferroelectric thin films. Phys. Rev. Lett. 104, 207602 (2010)

31 Balke, N., Choudhury, S., Jesse, S., Huijben, M., Chu, Y. H., Baddorf, A. P., Chen, L. Q. Ramesh, R. \& Kalinin, S. V. Deterministic control of ferroelastic switching in multiferroic materials. Nat. Nanotechnol. 4, 868-875 (2009).

32 Vasudevan, R. K., Morozovska, A. N., Eliseev, E. A., Britson, J., Yang, J.-C., Chu, Y.-H., Maksymovych, P., Chen, L. Q., Nagarajan, V. \& Kalinin, S. V. Domain wall geometry controls conduction in ferroelectrics. Nano Lett. 12, 5524-5531 (2012).

33 Yang, C.-H., Seidel, J., Kim, S. Y., Rossen, P. B., Yu, P., Gajek, M., Chu, Y. H., Martin, L. W., Holcomb, M. B., He, Q., Maksymovych, P., Balke, N., Kalinin, S. V., Baddorf, A. P., Basu, S. R., Scullin, M. L. \& Ramesh, R. Electric modulation of conduction in multiferroic Ca-doped $\mathrm{BiFeO}_{3}$ films. Nat. Mater. 8, 485-493 (2009).

34 Yang, C.-H., Kan, D., Takeuchi, I., Nagarajan, V. \& Seidel, J. Doping $\mathrm{BiFeO}_{3}$ approaches and enhanced functionality. Phys. Chem. Chem. Phys. 14, 15953-15962 (2012)

35 Chu, Y. H., Zhan, Q., Yang, C.-H., Cruz, M. P., Martin, L. W., Zhao, T., Yu, P., Ramesh, R., Joseph, P. T., Lin, I. N., Tian, W. \& Schlom, D. G. Low voltage performance of epitaxial $\mathrm{BiFeO}_{3}$ films on $\mathrm{Si}$ substrates through lanthanum substitution. Appl. Phys. Lett. 92, 102909 (2008). 
36 Lee, Y.-H., Wu, J.-M. \& Lai, C.-H. Influence of La doping in multiferroic perperties of $\mathrm{BiFeO}_{3}$ thin films. Appl. Phys. Lett. 88, 042903 (2006).

37 Chen, W., Ren, W., You, L., Yang, Y., Chen, Z., Qi, Y., Zou, X., Wang, J., Sritharan, T., Yang, P., Bellaiche, L. \& Chen, L. Domain structure and in-plane switching in a highly strained $\mathrm{Bi}_{0.9} \mathrm{Sm}_{0.1} \mathrm{FeO}_{3}$ film. Appl. Phys. Lett. 99, 222904 (2011). (c) (1) (2) $\odot$ This work is licensed under a Creative Commons Attribution-NonCommercial-NoDerivs 3.0 Unported License. To view a copy of this license, visit http://creativecommons. org/licenses/by-nc-nd/3.0/

Supplementary Information accompanies the paper on the NPG Asia Materials website (http://www.nature.com/am) 